\title{
The Effect of Passive Movement for Paretic Ankle-Foot and Brain Activity in Post-Stroke Patients
}

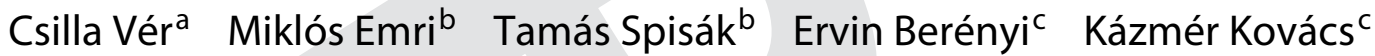 \\ Péter Katona $^{d}$ László Balkay ${ }^{b}$ László Menyhárt ${ }^{\mathrm{e}} \quad$ László Kardos $^{f}$ László Csiba ${ }^{a}$ \\ ${ }^{a}$ Department of Neurology, ${ }^{b}$ Department of Nuclear Medicine, and ${ }^{\mathrm{c}}$ Department of Medical Laboratory and \\ Imaging Sciences, University of Debrecen, dDepartment of Diagnostic Radiology, Kenézy Gyula County Hospital, \\ e Medical Systems Hungary Ltd., and ${ }^{\mathrm{f}}$ Medical Statistician, Kenézy Gyula County Hospital, Debrecen, Hungary
}

\section{Key Words}

Stroke · Ankle · Passive movement · Equinovalgus · fMRI

\begin{abstract}
Background: This study aims at investigating the short-term efficacy of the continuous passive motion (CPM) device developed for the therapy of ankle-foot paresis and to investigate by $\mathrm{fMRI}$ the blood oxygen level-dependent responses (BOLD) during ankle passive movement (PM). Methods: Sixty-four stroke patients were investigated. Patients were assigned into 2 groups: 49 patients received both 15 min manual and 30 min device therapy $(M+D)$, while the other group $(n=15)$ received only 15 min manual therapy (M). A third group of stroke patients $(n=12)$ was investigated by fMRI before and immediately after $30 \mathrm{~min}$ CPM device therapy. There was no direct relation between the fMRI group and the other 2 groups. All subjects were assessed using the Modified Ashworth Scale (MAS) and a goniometer. Results: Mean MAS decreased, the ankle's mean plantar flexion and dorsiflexion passive range of motion (PROM) increased and the equinovalgus improved significantly in the $M+D$ group. In the $\mathrm{FMRI}$ group, the PM of the paretic ankle increased BOLD responses; this was observed in the contralateral pre- and postcentral gyrus, superior temporal gyrus, central opercu-
\end{abstract}

lar cortex, and in the ipsilateral postcentral gyrus, frontal operculum cortex and cerebellum. Conclusion: Manual therapy with CPM device therapy improved the ankle PROM, equinovalgus and severity of spasticity. The ankle PM increased ipsi- and contralateral cortical activation.

(c) 2016 S. Karger AG, Basel

\section{Introduction}

Common ankle-foot deformities following stroke are flexible equinovalgus deformities. Equinovalgus deformity is a consequence, on the one hand, of spasticity in the gastrocnemius, soleus and peroneus brevis muscles and, on the other hand, of a loss of strength in the anterior and posterior tibial muscles, which impedes walking and balance, and contributes directly to functional limitations $[1,2]$.

Common methods in everyday practice for spasticity relief, deformity prevention, and improvement of gait safety include botulinum toxin treatment, the use of ankle-foot orthoses, and passive movement (PM) therapy. An injection of botulinum toxin into the gastrocnemius and soleus muscles does alleviate spasticity, but it also reduces muscle strength during the gait cycle.

\section{KARGER}

E-Mail karger@karger.com

www.karger.com/ene
C 2016 S. Karger AG, Basel

0014-3022/16/0000-0000\$39.50/0
Csilla Vér

Department of Neurology, University of Debrecen

Móricz Zsigmond Street 22

HU-4032 Debrecen (Hungary)

E-Mail vercsilla10@gmail.com 
Table 1. Clinical data of stroke patients in the manual $(M)$ and device $(M+D)$ groups

\begin{tabular}{llllll}
\hline & $\begin{array}{l}\text { Female/male, } \\
\text { subjects }\end{array}$ & $\begin{array}{l}\text { Ischemic/hemorrhagic } \\
\text { stroke, subjects }\end{array}$ & $\begin{array}{l}\text { Moderate/severe } \\
\text { paresis, subjects }\end{array}$ & $\begin{array}{l}\text { NIHSS, points, } \\
\text { mean } \pm \text { SD }\end{array}$ & $\begin{array}{l}\text { Age, years, } \\
\text { mean } \pm \text { SD }\end{array}$ \\
\hline M group & $7 / 8$ & $11 / 4$ & $8 / 7$ & $13.33 \pm 5.92$ & $71.3 \pm 10.39$ \\
M + D group & $18 / 31$ & $37 / 12$ & $20 / 29$ & $11.28 \pm 4.44$ & $62.2 \pm 12.38$ \\
\hline
\end{tabular}

For the period before voluntary ankle dorsiflexion ability is restored, a possible approach is the use of an ankle-foot orthosis; while this will produce a more stable and safer gait, it is insufficient on its own to induce the desired active motion [3].

One of the most widespread methods to induce active motion is continuous PM therapy. PM probably activates the sensorimotor system and helps by preventing deep venous thrombosis, improving the motor function, biomechanical and functional properties of the ankle after stroke $[4,5]$. The physiotherapist usually performs PM manually.

The number of physiotherapists available at stroke departments is limited; PM is tiresome for the therapist, and the intensity of movement may decrease after a number of repetitions. This may reduce the efficacy of PM therapy. That is why continuous passive motion (CPM) devices are so important [6]. These devices move the given joint in a specified motion range, with specified intensity, approaching the pain threshold. Using them may prevent the development of deep venous thrombosis or contractures, resolve spasticity by facilitating active exercise and sending stimuli to the cerebral cortex on a continuous basis during the PM therapy.

An electronic device can partially replace the physiotherapist if the strength, speed and frequency of movement are individually adaptable to the needs of the patient (severity of spasticity, etc.). In addition, the device should be easily transportable and safe, and last but not least, the efficacy of the therapy should be capable of being quantitatively measured.

Selected studies report the improvement of ankle joint parameters upon treatment with feedback-controlled passive stretching devices [3].

$\mathrm{Wu}$ et al. [7] combined active motion therapy with feedback-controlled stretching of the ankle joint in children with cerebral palsy, resulting in a significant improvement of the ankle joint's biomechanical and functional parameters.

Techniques such as functional magnetic resonance imaging are frequently used to measure the changes in the blood oxygen level-dependent (BOLD) magnetic reso- nance signal [8] in the investigation of the relationship between neural reorganization and functional recovery after stroke [9]. In stroke rehabilitation, post-stroke cerebral reorganization can be characterized by fMRI, using ankle-foot active movement, PM, and therapy [10-17].

In this study, we developed an electronic CPM device, and assessed the clinical improvement in terms of angle, passive range of motion (PROM), equinovalgus, and spasticity of patients treated with the device [2]. We investigated if $30 \mathrm{~min}$ device therapy with $15 \mathrm{~min}$ manual therapy was as effective as 15 min manual therapy. In addition we also investigated using fMRI whether the passive motion evokes acute BOLD changes in the ipsi- and/ or contralateral hemisphere.

\section{Materials and Methods}

Our Clinical Department is an acute stroke treatment center; immediately upon stabilization of condition, patients are transferred to a rehabilitation institute, where they receive complex rehabilitation (physiotherapy, ergotherapy, speech therapy, psychotherapy). Patients are treated at our center for an average of 7 days, and therefore, the aim was to study the short-term efficacy of the CPM therapy.

\section{Subjects}

Manual Group (M) and Device Group (M+D)

Sixty-four acute ischemic and hemorrhagic stroke patients with impaired ankle-foot motor function were investigated. $\mathrm{Pa}$ tients were assigned to 2 groups consecutively: one group $(\mathrm{n}=49$; mean age \pm SD: $62.2 \pm 12.38$ years; female to male ratio: 18 to 31 ) received manual therapy combined with 30 min device therapy $(\mathrm{M}+\mathrm{D})$ for 1 week, and the manual group $(\mathrm{M})(\mathrm{n}=15$; mean age \pm SD: $71.3 \pm 10.39$ years; female to male ratio: 7 to 8 ) received only 15 min manual therapy for 1 week by a physiotherapist.

We had no influence on the sequence of patients.

Manual and device-based therapy were started 24-48 h after stroke onset [2].

Patients' demographic and pathological data are summarized in table 1 .

Functional MRI Group (fMRI Group)

A third group of ischemic stroke patients $(n=12$; mean \pm SD) time of stroke: $56.08 \pm 133.88$ days; mean \pm SD age: $65.3 \pm 9.59$ years; female to male ratio: 7 to 5 were treated by our device on a 
Table 2. Changes (absolute difference; relative improvement) after therapy in the $M$ and $M+D$ groups, with reference to baseline values, in: PROM (from maximum plantar to maximum dorsiflexion), degree of equinovalgus deformity, and MAS score

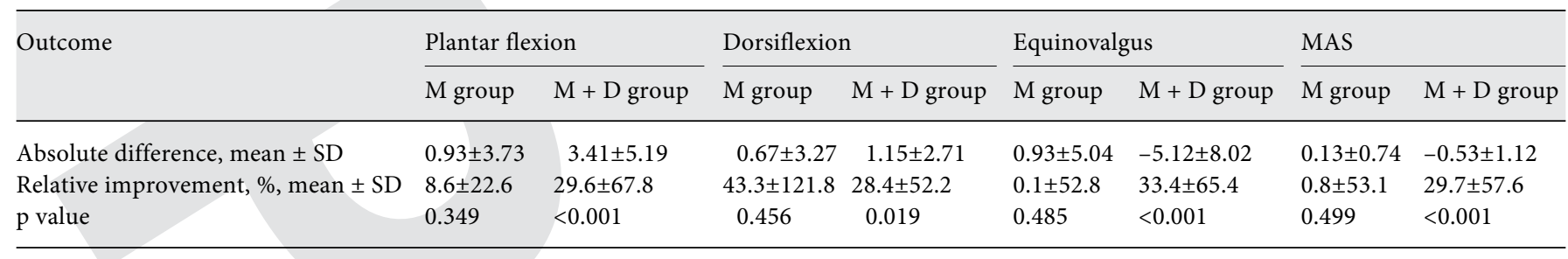

single occasion and investigated by fMRI (fMRI group) before and immediately after 30 min device therapy. Demographic data, pathology data and clinical characteristics of fMRI group (ankle passive ranges of motion, scores of National Institute of Health Stroke Scale (NIHSS) and Modified Ashworth Scale (MAS)) are summarized in table 3 . There was no direct relation between the patient group examined by $\mathrm{fMRI}$ and the $2(\mathrm{M}+\mathrm{D}$ and $\mathrm{M}$ groups) other patient groups.

Inclusion and Exclusion Criteria of Manual (M), Device (M + D) and fMRI Groups

The inclusion criteria for the $\mathrm{M}, \mathrm{M}+\mathrm{D}$, and $\mathrm{fMRI}$ groups were ischemic and/or hemorrhagic stroke confirmed via clinical investigations and CT.

Patients suffered from mild, moderate or severe lower limb paresis (item 6 of the NIHSS $\geq 1$ point) due to ischemic or hemorrhagic stroke [18].

Patients were excluded from participation if any of the following criteria were met: primarybrain tumor, brain metastasis, Parkinson's disease, multiple sclerosis, rheumatoid arthritis, ankylosis of the ankle and foot joints, trauma or surgery of the foot and ankle; and patients with psychiatric symptoms were also excluded. The study was approved by the Regional and Institutional Ethics Committee; written informed consent was obtained from each patient.

\section{The Device}

Currently in the prototype phase, the passive motion device works by mobilizing the paretic foot in a repeated fashion across the ankle's entire range of motion (ROM), from plantar to dorsiflexion in one plane. Weighing only $7 \mathrm{~kg}$ and measuring 25 by 52 by $45 \mathrm{~cm}$, the device is designed for optimum portability and usability at the bedside. Actuation is provided by a 24 -volt direct current motor. Operating frequency limits include 7 cycles per minute from plantar to dorsiflexion (0-60 degrees) at the high, and $4 \mathrm{cy}$ cles per minute from plantar to dorsiflexion at the low end (fig. 3).

Resistance parameters were mostly set between 1 and $7 \mathrm{Nm}$ of maximum resistance torque in dorsiflexion, and 1-10 Nm maximum resistance torque in plantar flexion. Motion parameters (angle, power, speed) can be individualized to suit the patient's level of tolerance and pain threshold. The device has a built-in mechanism to stop automatically upon the detection of active resistance. During the study, no cases of either the extension or the flexor motion provoking any pain have been observed. The daily session length of device-based therapy was $30 \mathrm{~min}$; the average total treatment period was 5.5 days (range 4-7) in the reference (patients receiving standard manual physiotherapy only) and the $M+D$ groups alike [2].

Effect of PM for Paretic Ankle-Foot and Brain Activity
Neurological Status and Lower Limb Measurements

All patients were assessed using the MAS, NIHSS and goniometer measurements (tables 1 and 3).

Since the soleus muscle plays an important role in the foot's plantar flexion, and also in maintaining a stable stance, we assessed the level of spasticity in this muscle using the MAS, a quick and easy-to-use tool to evaluate spasticity.

During soleus muscle tone assessment, the patient was relaxing in a supine position, with their head in the midline and arms close to the trunk. The procedure is designed to maintain the knee joint in a slight flexion between 0 and 45 degrees, thereby isolating soleus functions from the unwanted addition of gastrocnemius muscle work. By placing one hand on the patient's foot and another on the area above the ankle joint, the examiner performed the PM of the ankle joint toward plantar and dorsal flexion. This was repeated 3 times in total [19].

The point score definitions of the MAS are:

0 points: no increase in muscle tone,

1 point: slight increase in muscle tone, manifested by a catch and release or by minimal resistance at the end of the ROM when the affected part(s) is moved in flexion or extension,

2 points: slight increase in muscle tone, manifested by a catch, followed by minimal resistance throughout the remainder (less than half) of the ROM,

3 points: more marked increase in muscle tone through most of the ROM, but affected part(s) easily moved,

4 points: considerable increase in muscle tone, $\mathrm{PM}$ difficult, 5 points: affected part(s) rigid in flexion or extension $[2,20]$.

We used the NIHSS scale to determine the functional state and the severity of the stroke in our patients. NIHSS is one of the widest spread scales in the clinical field; increasing scores indicate more severe functional states of the patients.

The goniometer was used for measuring the ROM (in degrees) of passive ankle plantar or dorsiflexion, and equinovalgus deformity before and after treatment. The PROM assessment procedure was performed with the ankle in a neutral position (90-degree angle between leg and foot), the knee fully extended, and the hip in a neutral position (i.e. not in rotation; fig. 1).

The extent of the equinovalgus deformity was also determined using goniometer. During the measuring of deformity, the patient was in a supine position, with the knees fully extended and the hip joint in neutral position. Measuring was performed with the patient's leg fixed by straps. The pivot point of the angle gauge fell in the middle of the patient's heel bone. The adjustable bumper of the goniometer was set individually to the patient's deformed foot, more closely, to the distal phalanx of the fifth toe; that was the starting point, that is, ' 0 degrees'. The examiner moved the foot from 


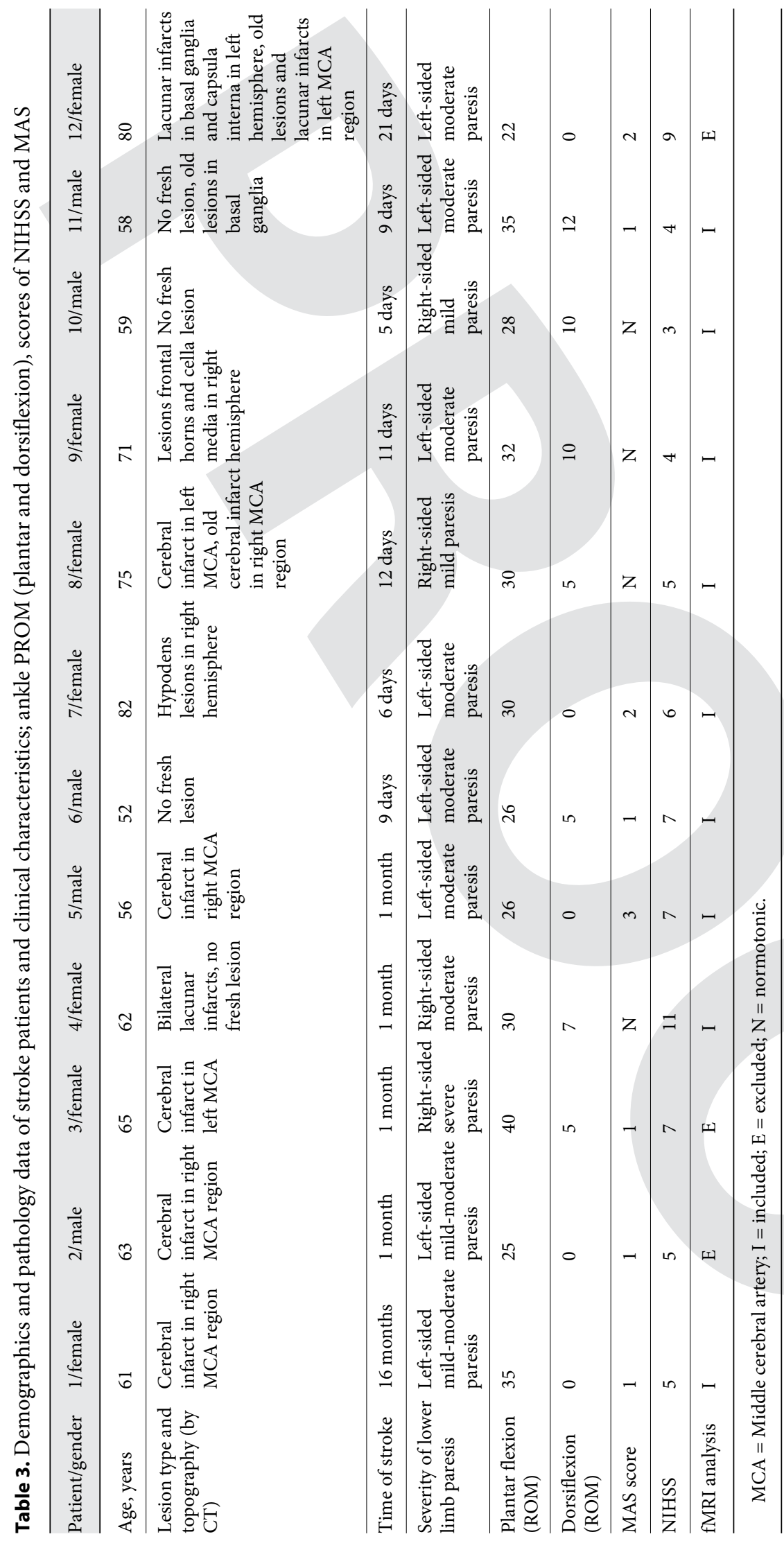




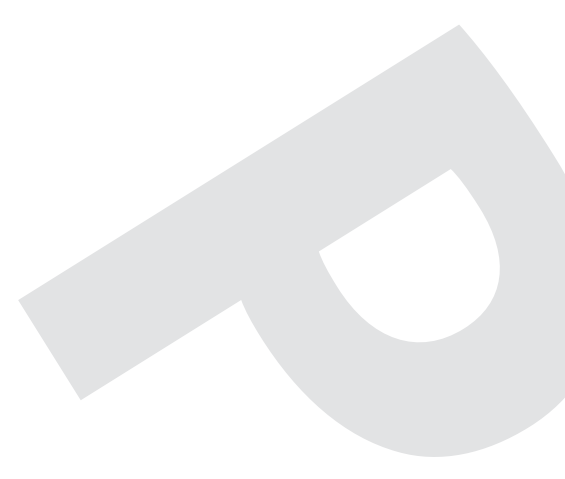

Fig. 1. Goniometer measurement of plantar and dorsiflexion ranges. 'ROM' is a term for the radius of motion achieved by external manipulation without the patient using their own muscles.
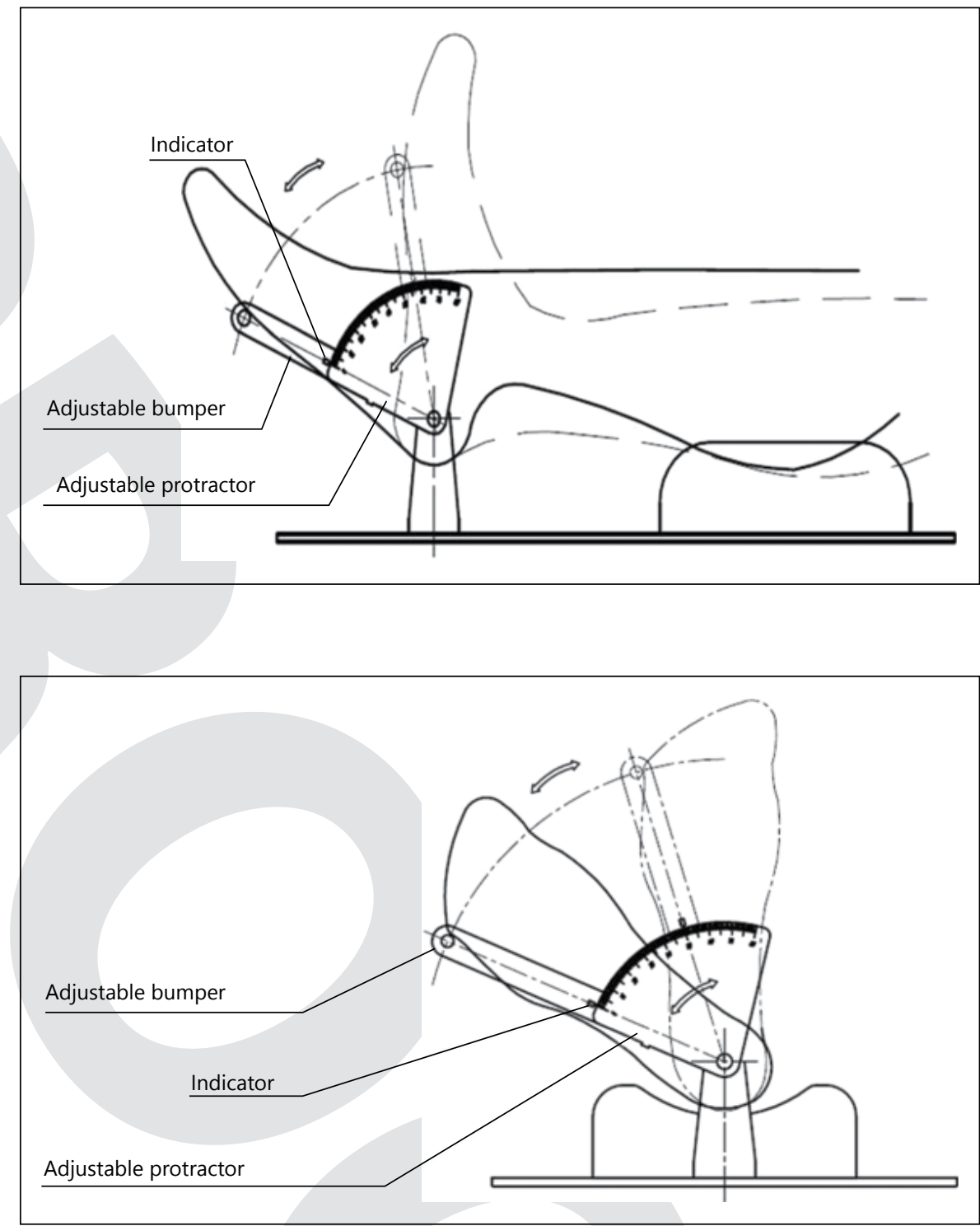

Fig. 2. Goniometer measurement of the extent of the equinovalgus deformity of the foot.

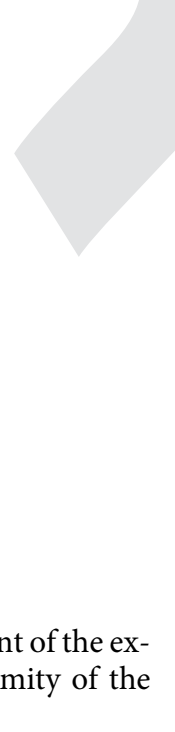

this setting to the vertical physiological position, following the motion of the foot with the adjustable bumper of the goniometer in the meantime. The extent of deformity can be read from the goniometer with the leg in the vertical position (fig. 2).

The active ROM of the ankle joint was not evaluated because mental confusion and difficulty of communication (aphasia and/ or dementia) present during acute stroke are known to substantially impede or bias the accurate assessment of active ROM.

\section{Investigation of the fMRI Group}

Image Acquisition

Images were acquired at Kenézy Hospital, Debrecen using a 1.5 tesla Siemens Magnetom Essenza magnetic resonance scanner. A 3D T1-weighted axial magnetization-prepared rapid acquisition with gradient echo structural image was obtained (echo time $(\mathrm{TE})=4.73 \mathrm{~ms}$, repetition time $(\mathrm{TR})=1,540 \mathrm{~ms}$, inversion time $=$ $800 \mathrm{~ms}$, flip angle $=15$ slices with $0.9 \times 0.9 \times 0.9 \mathrm{~mm}$ voxels $)$. Func- tional images were obtained using a BOLD contrast sensitive gradient-echo echo-planar sequence $(\mathrm{TE}=42 \mathrm{~ms}$, flip angle $=90$, inplane resolution $=3 \times 3 \mathrm{~mm}$; volume $\mathrm{TR}=4,000 \mathrm{~ms}$ ). Whole-brain coverage for the functional data was obtained using 41 contiguous interleaved 3-mm axial slices. Each functional session consisted of 100 functional volumes.

\section{Functional MRI}

Before fMRI investigation, a goniometer was used to measure the ROM of passive ankle plantar and dorsiflexion. During the 400-second-long functional MRI sessions, 40-second-long active and passive blocks were employed (a total of 10 block pairs, beginning with a passive block). In the passive block, no stimulus was applied, whereas in the active block, slow CPM of the left or right foot was carried out by the physiotherapist for $40 \mathrm{~s}$. The effects of the PM of the left versus the right foot were investigated in separate sessions. 


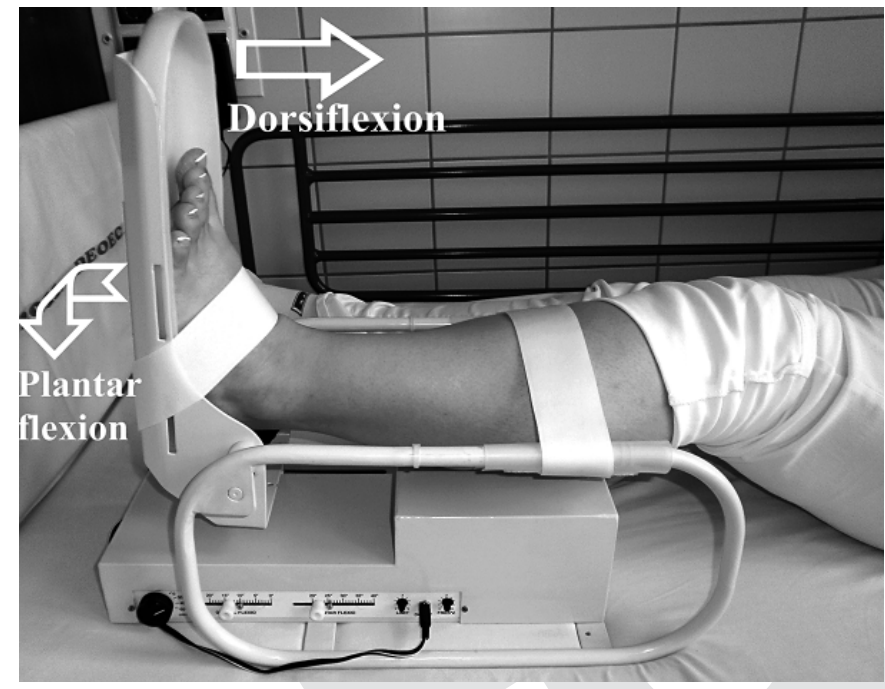

Fig. 3. The device.

For each subject, 2 fMRI investigations for both the left and right foot movement paradigm were applied. Between these measurements, 30-minute session of CPM was performed on the paretic leg by our passive motion device. Angle, power and speed parameters were individually adapted to the patients during therapy.

Image Processing

Before preprocessing, the left and the right sides of the structural and functional images of patients with left-hemispheric lesion were swapped, so that in all of the images, the lesioned side was toward the positive direction on the $\mathrm{x}$-axis (right side of the image in neurological convention). This step allowed us to conduct a pooled population-level statistical analysis for all of the patients, and avoid splitting the population into 2 cohorts based on the side of the stroke.

The fMRI time series were motion corrected using the intramodal motion correction utility of the functional MRI of the brain software library (FSL) [21,22]. The first 3 volumes of each data set were discarded from further analysis to allow for T1 equilibration effects. The brain extraction utility of FSL was used to remove nonbrain areas from both the functional and structural scans [23]. For each fMRI session, a 'noise region' was delineated by the analysis of the temporal signal-to-noise ratio of BOLD signals, and 5 principal noise components were extracted from the data following the component-based noise correction method [24].

The resulting preprocessed fMRI data were non-linearly coregistered to the brain-extracted anatomical image and then spatially transformed to the symmetric template of Montreal Neurological Institute (MNI) 152 space [25] using linear and non-linear registration utilities of the FSL package [26], to achieve spatial correspondences for group analysis.

\section{Statistical Analysis}

Statistical Analysis of the Manual (M) and Device (M + D)

Groups

Within-subject changes were calculated as the difference between pre-session readings at the last available session and baseline readings. Such changes were tested for significance using Student's paired $\mathrm{t}$ tests (if normality assumptions were satisfied) or Wilcoxon's matched-pairs signed-ranks tests (if normality assumptions failed). The statistical package Stata was used for data analysis $[2,27]$.

\section{Statistical Analysis of fMRI Group}

A total of 12 patients were included in the fMRI group in compliance with the selection criteria; however, 2 patients had to be excluded from the statistical analysis: one due to claustrophobia, which developed in the course of MRI, and the other due to extreme head movements related to stimuli received during the fMRI recording.

Before statistical analysis, an isotropic Gaussian smoothing with $8-\mathrm{mm}$ full width at half maximum was applied to the functional images. Data were then analyzed within a general linear model (GLM) framework. In the voxel-wise GLM-based first-level analysis, we incorporated 7 explanatory variables: the hypothesis for the stimulus according to the block design, the temporal derivate thereof (to allow for slight variations in timing), and the 5 principal noise component time courses based on the noise correction analysis. We convolved the predictor of interest with a doublegamma hemodynamic response function.

The resulting statistical parametric maps were analyzed on the population level with 2 second-level, fixed-effect models corresponding to the healthy (non-paretic) ankle CPM (hCPM) and paretic ankle CPM (pCPM) stimulations denoted respectively by hCPM and pCPM. In line with the paired-sample design, withinsubject variance was modeled with a factor variable encoding the session repetition, that is, whether the scan was taken before or after the CPM therapy. Four population-level statistical parametric images were constructed according to both stimuli and 2 sessions: 2 mean activations of hCPM and pCPM evaluated from first-session fMRI data (table 4) and 2 increased activation maps calculated by a 2 -sample paired t test using repeated measurements (table 5). Statistical parametric images were corrected for multiple comparison using Gaussian Random Field theory [28] and cluster probability thresholding [29]. Clusters were defined by a $\mathrm{z}$-value threshold of 3.1 ( $\mathrm{p}_{\text {uncorrected }}<0.001$ ), and activation clusters having a $p$ value $<0.05$ were discarded. The emphasized activation clusters were identified by the atlas toolbox of FSL view software and the database of Harvard-Oxford cortical and subcortical structural atlases [30].

\section{Results}

\section{Manual Group (M)}

The changes (absolute difference and relative improvement) in plantar flexion, dorsiflexion, equinovalgus and MAS score with reference to baseline values in the $M$ and $\mathrm{M}+\mathrm{D}$ groups are shown in table 2 .

No significant changes could be detected in the ankle plantar flexion $(\mathrm{p}=0.349)$ and dorsiflexion degrees $(\mathrm{p}=$ 0.456).

The improvement of equinovalgus deformity ( $\mathrm{p}=$ $0.485)$ and MAS ( $\mathrm{p}=0.499)$ were not significant (table 2). 
Table 4. Brain regions significantly activated by hCPM and pCPM stimulation

\begin{tabular}{|c|c|c|c|c|c|c|c|c|c|c|}
\hline & $\begin{array}{l}\text { Cluster } \\
\text { index }\end{array}$ & $\mathrm{cm}^{3}$ & $\mathrm{p}$ value & $-\log _{10}(\mathrm{p})$ & $\mathrm{z}_{\max }$ & $\mathrm{x}, \mathrm{mm}$ & $\mathrm{y}, \mathrm{mm}$ & $\mathrm{z}, \mathrm{mm}$ & $\mathrm{I} / \mathrm{C}$ & Region \\
\hline \multirow{5}{*}{$\begin{array}{l}\text { Healthy } \\
\text { ankle }\end{array}$} & 1 & 12.40 & $1.99 \times 10^{-8}$ & 7.70 & 15.00 & -3 & -30 & 69 & $\mathrm{C}$ & Precentral gyrus (M1) \\
\hline & & & & & 14.60 & -9 & -42 & 78 & $\mathrm{C}$ & Postcentral gyrus (S1) \\
\hline & & & & & 9.40 & -12 & -53 & 75 & $\mathrm{C}$ & Superior parietal lobule \\
\hline & & & & & 7.00 & -45 & -31 & 22 & $\mathrm{C}$ & Parietal operculum cortex (S2) \\
\hline & 3 & 6.40 & $2.53 \times 10^{-5}$ & 4.60 & 8.100 & 68 & -25 & 18 & I & pSTG \\
\hline \multirow{3}{*}{$\begin{array}{l}\text { Paretic } \\
\text { ankle }\end{array}$} & 1 & 9.80 & $2.49 \times 10^{-8}$ & 5.55 & 16.60 & 8 & -37 & 78 & C & Postcentral gyrus (S1) \\
\hline & & & & & 13.20 & 6 & -28 & 76 & $\mathrm{C}$ & Precentral gyrus (M1) \\
\hline & & & & & 4.200 & -55 & -31 & 27 & $\mathrm{I}$ & Parietal operculum cortex (S2) \\
\hline
\end{tabular}

Activation clusters (bold lines) were emphasized by a $\mathrm{z}$-value threshold of 3.1 and $\mathrm{p}$ value $<0.05$ using random field theory. Each cluster is reported by its extent $\left(\mathrm{cm}^{3}\right)$, obtaining probability ( $\mathrm{p}$ and $\left.-\log _{10}(\mathrm{p})\right)$ and localization parameters of its maximal activated voxel: $\mathrm{z}$-value $\left(\mathrm{z}_{\max }\right)$, location in MNI152 space $(\mathrm{x}(\mathrm{mm}), \mathrm{y}(\mathrm{mm}), \mathrm{z}(\mathrm{mm}))$, lateralization (I/C i.e. ipsi-, contralateral) and the atlas region identified by Harvard-Oxford cortical and subcortical structural atlases. Local maxima within cluster occurred in different atlas regions are also reported.

Table 5. Brain regions show significantly higher activation in the second session by hCPM and pCPM stimulation

\begin{tabular}{|c|c|c|c|c|c|c|c|c|c|c|}
\hline & $\begin{array}{l}\text { Cluster } \\
\text { index }\end{array}$ & $\mathrm{cm}^{3}$ & $\mathrm{p}$ value & $-\log _{10}(p)$ & $\mathrm{z}_{\max }$ & $\mathrm{x}, \mathrm{mm}$ & $\mathrm{y}, \mathrm{mm}$ & $\mathrm{z}, \mathrm{mm}$ & $\mathrm{I} / \mathrm{C}$ & Region \\
\hline \multirow{2}{*}{$\begin{array}{l}\text { Healthy } \\
\text { ankle }\end{array}$} & 1 & 2.50 & 0.0413 & 1.38 & 4.190 & 4 & 42 & 44 & I & SFG \\
\hline & & & & & 4.150 & 7 & 37 & 41 & I & SFG/paracingulate gyrus \\
\hline \multirow{5}{*}{$\begin{array}{l}\text { Paretic } \\
\text { ankle }\end{array}$} & 1 & 6.70 & 0.00515 & 2.29 & 5.63 & 20 & -11 & -36 & I & aPHG \\
\hline & & & & & 5.75 & 49 & -17 & -30 & I & pITG \\
\hline & & & & & 4.57 & 33 & -10 & -47 & I & $\begin{array}{l}\text { Temporal fusiform cortex, } \\
\text { posterior division/temporal } \\
\text { fusiform cortex, anterior division }\end{array}$ \\
\hline & 2 & 4.60 & 0.021 & 1.68 & 5.040 & -29 & -15 & -29 & C & aPHG \\
\hline & & & & & 3.80 & -53 & -20 & -24 & C & pITG \\
\hline
\end{tabular}

Activation clusters (bold lines) were emphasized by a $\mathrm{z}$-value threshold of 3.1 and $\mathrm{p}$ value $<0.05$ using random field theory. Each cluster is reported by its extent $\left(\mathrm{cm}^{3}\right)$, obtaining probability ( $\mathrm{p}$ and $-\log _{10}(\mathrm{p})$ ) and localization parameters of its maximal activated voxel: $\mathrm{z}$-value $\left(\mathrm{z}_{\max }\right)$, location in MNI152 space $(\mathrm{x}(\mathrm{mm}), \mathrm{y}(\mathrm{mm}), \mathrm{z}(\mathrm{mm}))$, lateralization (I/C i.e. ipsi-, contralateral) and the atlas region identified by Harvard-Oxford cortical and subcortical structural atlases. Local maxima within cluster occurred in different atlas regions are also reported.

Effect of PM for Paretic Ankle-Foot and Brain Activity 

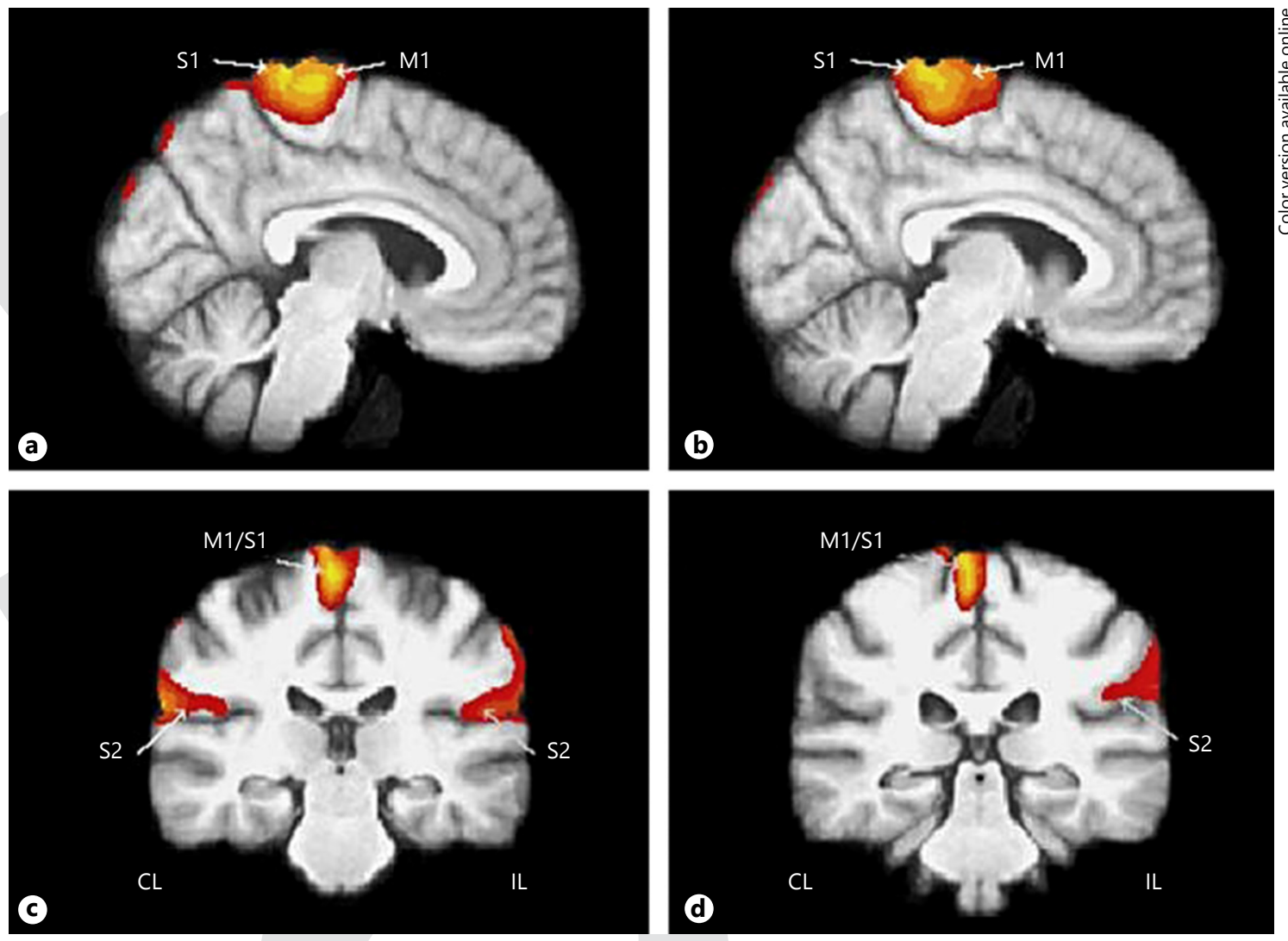

Fig. 4. Significantly activated brain regions during hCPM and pCPM stimulation superimposed on averaged T1-weighted images of the investigated population. Both stimuli-activated regions in the contralateral (CL) primary motor (M1) and primary somatosensory (S1) cortex (a, b). The peak activities of S1 and
M1 areas were almost equal in the case of hCPM in contrast to pCPM (arrows on $\mathbf{a}$ and $\mathbf{b}$ ). Regions in secondary somatosensory cortex (S2) connected with other areas were activated ipsilaterally (IL) in both cases and contralaterally in the case of hCPM stimuli $(\mathbf{c}, \mathbf{d})$.
Device Group $(M+D)$

The ankle's mean plantar flexion range increased significantly by 3.41 (SD 5.19) degrees $(\mathrm{p}<0.001)$ and the dorsiflexion also improved significantly by 1.15 (SD 2.71) degrees $(\mathrm{p}=0.019)$.

The mean flexible equinovalgus deformity improved by -5.12 (SD 8.02) degrees ( $\mathrm{p}<0.001)$.

The MAS score decreased by $0.53(1.12)$ points $(\mathrm{p}<$ $0.001)$.

The results suggest that manual therapy combined with the device therapy had a beneficial effect, while the short-term manual therapy alone was not effective.

\section{Results of PM Stimuli in the fMRI Group}

Analysis was performed on the block design fMRI data of 10 patients, because one of the patients did not cooperate during the scan due to claustrophobia, and another was excluded from the statistical analysis due to extreme in-scanner head displacement and stimulus-correlated movement (table 3 ).

In the statistical analysis of first-session (pre-training) data, we found that both the hCPM and pCPM stimuli activated the contralateral primary motor (M1) and primary somatosensory (S1) cortex (table 4; fig. 4a and b). Furthermore, we observed activated voxels (with $\mathrm{z}=7.0$ maximal value) in the small part of the supplementary motor area (SMA) during the PCPM in contrast to hCPM.

Overlapping activity in the anterior division of the supramarginal gyrus (aSMG) and secondary somatosensory/parietal operculum cortex (S2) was found contralaterally in the case of hCPM (table 4), and ipsilaterally during pCPM (table 4; fig. 4c and d).

In the case of hCPM, a third ipsilateral activation cluster was also detected, overlapping the part of the posterior division of the superior temporal gyrus and insular region of S2 (table 4; fig. 4c). 

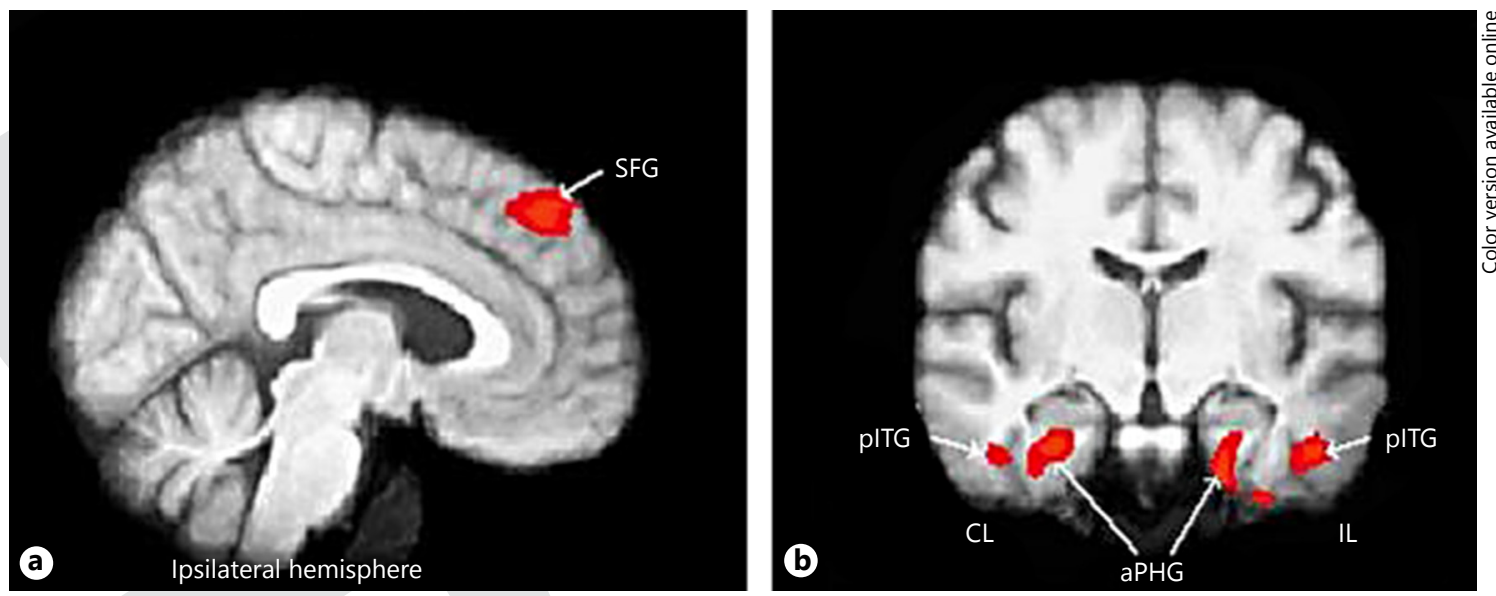

Fig. 5. Brain regions - superimposed on averaged T1-weighted images of the investigated population - where the activation was significantly higher in the second session than in the first. a, b Non-significant activation change in the primary motor (M1), and primary and secondary somatosensory (S1 and S2) cortices.
In the case of hCPM, we detect significantly higher activity in the second session only in the SFG in the ipsilateral hemisphere. The pCPM showed significantly higher activity in the second session bilaterally in the aPHG connected with the area of the pITG.
Comparing data from 2 fMRI sessions (before and after treatment) revealed increased activation in the post-treatment session both for hCPM and PCPM (table 4). In the case of hCPM, we found only one cluster in the superior frontal gyrus (SFG) near the cluster-significance threshold ( $\mathrm{p}_{\text {cluster }}<0.0413$ ) with a size of 2.5 $\mathrm{cm}^{3}$, and $\mathrm{z}=4.1$ peak activity (table 5; fig. 5a). During the pCPM, 2 bilaterally located clusters were observed (table 5; fig. 5b) in the anterior division of the parahippocampal gyrus $(\mathrm{aPHG})$ connected to the activated area of the posterior division of the inferior temporal gyrus (pITG).

\section{Discussion}

First, our study demonstrates that our device significantly improves the PROM of the ankle and other clinical parameters. Second, therapy with our device appears to be more efficient than manual therapy [2]. Third, we proved that therapy with our device resulted not only in clinical improvement but also in acute BOLD-response changes after the therapeutic session.

Our research group developed a low-cost, practical device that moves the ankle joint in plantar flexion and dorsiflexion directions safely [2]. Compared to the ankle motion devices mentioned in the literature and tested with stroke patients, our device is light and portable, and treatment can be carried out on the patient's bed [31-33]. This is very important in the case of stroke with bleeding, or unstable cases of internal medicine.

In the patient group where we performed manual therapy using our passive motion device $(\mathrm{M}+\mathrm{D}), \mathrm{PROM}$ improved, and the extent of spasticity decreased, as did the degree of equinovalgus deformity [5].

The $\mathrm{M}+\mathrm{D}$ group showed significant improvement in the MAS score; however, the observed MAS score reduction of 0.53 points in the $\mathrm{M}+\mathrm{D}$ group is arguably of very limited clinical significance since it is within the error margin of the scale [34].

PROM and deformities must be restored before any other treatment can be effective. The maximum ankle dorsiflexion ROM requirements to ascend and descend stairs ranged between averages of 14-27 and 24-31 degrees, respectively. Rising up from a sitting posture requires significant ankle dorsiflexion ROM. The full ankle plantar dorsiflexion ROM may be required when reaching out to get objects. In addition, the subtalar, transverse tarsal and forefoot joints must be fully mobile to allow the foot to adapt to altering degrees of broken ground. So the first goal of acute phase of rehabilitation is to prevent and correct PROM and flexible deformities to increase function [35].

We assume that the stimuli generated in the course of movement by the passive motion device are uniform, in contrast to movement performed by the physiotherapist. The frequency, intensity and motion range can be set, and the settings can be used the following day again. In addi- 
tion, there is no fatigue effect, and thus, the treatment is independent of the physiotherapist's physical or mental state or individual performance, which may change from day to day [31].

We found no study in the available literature that includes the use of an ankle passive motion device in addition to manual therapy and comparison of the 2 in cases of acute stroke patients.

We also studied the effect of therapy applied with the passive motion device on the hemodynamic responses that are related to the nervous activity of the brain. There are fMRI studies in the literature that address the effect of $\mathrm{PM}$ of the ankle joint on the nervous activity of the brain in healthy participants [36-39]; however, there is limited knowledge on the effect in stroke patients $[11,17]$.

Our results demonstrate the PM of a paretic anklegenerating activation in the contralateral S1, M1 and SMA areas, and the ipsilateral supramarginal gyrus and S2 area (table 4; fig. 4b and d). In contrast to our observation, Enzinger et al. [11] also described activation during paretic ankle PM in the contralateral sensorimotor cortex, but they did not report the differences between peak activations of S1 and M1. We have shown that the peak activity in M1 was lower than in S1 during pCPM in contrast to the hCPM where these values were almost equal.

We hypothesize that PM as sensory information (such as continuous tactile perception) and as a motor task contributes to motor learning and motor function. Additionally, it can facilitate active movement. In our study, the repetitions of fMRI scans in hCPM vs. pCPM differs: in the former case, the second session was simple repeated measurement, while in the latter case, 30-minute-long therapy was performed using our CPM device between the scans. This difference explains why the 2 increased activation patterns differ (fig. 5; table 5). We found increased activity in the SFG (MNI coordinates: $4,42,44$ ) in the repeated measurement compared to the first session during hCPM. This area was also reported by Zimmermann et al. [40] as a motor planning task-related activated area. The between-sessions therapy resulted in higher activity bilaterally in the aPHG. Adhikari et al. [41] investigated the neural basis of movement synchronization through a finger-tapping fMRI study. They described greater brain activity during tapping rate deceleration in the cerebellum, superior temporal gyrus and parahippocampal gyrus. We hypothesize that the detected increased activity in the aPHG after the mid-session training may be related to the motion synchronization learning process.
As far as we know, there are no similar studies on CPM devices in the available literature that tested acute stroke patients and evaluated the effect of PM on brain activity patterns by fMRI.

Limitations of our study include the lack of knowledge on how long the effect of the treatment should last. It remains to be seen whether further improvement might be expected with long-term treatment - there may also be a point beyond which the therapy may not have any additional effect. We do not know how permanent the changes in BOLD response are; this is the subject of a future longitudinal study.

In conclusion, this study shows that ankle-foot manual therapy combined with CPM device therapy significantly improves ankle PROM, decreases the equinovalgus of a paretic foot, and decreases the severity of spasticity in the acute phase of rehabilitation.

We tested a passive motion-based fMRI paradigm that could be suitable for further neuroimaging studies. Additionally, we concluded from our findings that PM of a paretic ankle influences both the ipsilateral and contralateral hemispheres.

\section{Acknowledgments}

We would like to thank and express our sincere appreciation to the Kenézy Gyula County Hospital, Department of Neurology and the Kenézy Gyula County Hospital, Department of Rehabilitation for encouraging their patients to participate in our study.

Special thanks to MRI radiographers at Kenézy Gyula County Hospital for their excellent technical assistance.

\section{Statement of Ethics}

This research has been approved by the local Ethic Committee, and complies with the Declaration of Helsinki about human experimentation.

\section{Disclosure Statement}

All authors declare that there are no conflicts of interest.

\begin{tabular}{|c|c|}
\hline References & $\begin{array}{l}1 \text { Morrell DS, Pearson JM, Sauser DD: Progres- } \\
\text { sive bone and joint abnormalities of the spine } \\
\text { and lower extremities in cerebral palsy. Ra- } \\
\text { diographics 2002;22:257-268. } \\
2 \text { Vér C, Hofgárt G, Menyhárt L, Kardos L, } \\
\text { Csiba L: Ankle-foot continuous passive } \\
\text { motion device for mobilization of acute } \\
\text { stroke patients. Open J Ther Rehabil 2015;3: } \\
\text { 23-34. }\end{array}$ \\
\hline
\end{tabular}

Vér/Emri/Spisák/Berényi/Kovács/Katona/ Balkay/Menyhárt/Kardos/Csiba 
3 Waldman G, Yang CY, Ren Y, Liu L, Guo X, Harvey RL, et al: Effects of robot-guided passive stretching and active movement training of ankle and mobility impairments in stroke. NeuroRehabilitation 2013;32:625-634.

4 Lindberg P, Schmitz C, Forssberg H, Engardt $\mathrm{M}$, Borg J: Effects of passive-active movement training on upper limb motor function and cortical activation in chronic patients with stroke: a pilot study. J Rehabil Med 2004;36: 117-123.

5 Szameitat AJ, Shen S, Conforto A, Sterr A: Cortical activation during executed, imagined, observed, and passive wrist movements in healthy volunteers and stroke patients. Neuroimage 2012;62:266-280.

6 Gao F: Development of motorized facilitated ankle stretching. 2010. http://www.asbweb. org/conferences/2010/abstracts/40.pdf (accessed December 12, 2014).

7 Wu YN, Hwang M, Ren Y, Gaebler-Spira D, Zhang LQ: Combined passive stretching and active movement rehabilitation of lower-limb impairments in children with cerebral palsy using a portable robot. Neurorehabil Neural Repair 2011;25:378-385.

8 Huettel SA, Song AW, McCarthy G: Functional Magnetic Resonance Imaging, ed 2. Massachusetts, Sinauer, 2007.

9 Calautti C, Naccarato M, Jones PS, Sharma N, Day DD, Carpenter AT, et al: The relationship between motor deficit and hemisphere activation balance after stroke: a 3T fMRI study. Neuroimage 2007;34:322-331.

10 Carey JR, Anderson KM, Kimberley TJ, Lewis SM, Auerbach EJ, Ugurbil K: fMRI analysis of ankle movement tracking training in subject with stroke. Exp Brain Res 2004;154:281290.

11 Enzinger C, Johansen-Berg H, Dawes H, Bogdanovic M, Collett J, Guy C, et al: Functional MRI correlates of lower limb function in stroke victims with gait impairment. Stroke 2008;39:1507-1513.

12 Enzinger $\mathrm{C}$, Dawes $\mathrm{H}$, Johansen-Berg $\mathrm{H}$, Wade D, Bogdanovic M, Collett J, et al: Brain activity changes associated with treadmill training after stroke. Stroke 2009;40:24602467.

13 Casellato C, Ferrante S, Gandolla M, Volonterio N, Ferrigno G, Baselli G, et al: Simultaneous measurements of kinematics and fMRI: compatibility assessment and case report on recovery evaluation of one stroke patient. J Neuroeng Rehabil 2010;7:49.

14 Jang SH, Ahn SH, Lee J, Cho YW, Son SM: Cortical reorganization of sensori-motor function in a patient with cortical infarct. NeuroRehabilitation 2010;26:163-166.
15 Jang SH: Motor recovery mechanisms in patients with middle cerebral artery infarct: a mini-review. Eur Neurol 2012;68:234-239.

16 Deng H, Durfee WK, Nuckley DJ, Rheude BS, Severson AE, Skluzacek KM, et al: Complex versus simple ankle movement training in stroke using telerehabilitation: a randomized controlled trial. Phys Ther 2012;92:197209.

17 Del Din S, Bertoldo A, Sawacha Z, Jonsdottir J, Rabuffetti M, Cobelli C, et al: Assessment of biofeedback rehabilitation in post-stroke patients combining fMRI and gait analysis: a case study. J Neuroeng Rehabil 2014;11:53.

18 Brott T, Adams HP Jr, Olinger CP, Marler JR, Barsan WG, Biller J, et al: Measurements of acute cerebral infarction: a clinical examination scale. Stroke 1989;20:864-870.

19 Blackburn M, van Vliet P, Mockett SP: Reliability of measurements obtained with the modified Ashworth scale in the lower extremities of people with stroke. Phys Ther 2002;82: 25-34.

20 Gorgey AS, Chiodo AE, Zemper ED, Hornyak JE, Rodriguez GM, Gater DR: Relationship of spasticity to soft tissue body composition and the metabolic profile in persons with chronic motor complete spinal cord injury. J Spinal Cord Med 2010;33:6-15.

21 Jenkinson M, Bannister P, Brady M, Smith S Improved optimization for the robust and accurate linear registration and motion correction of brain images. Neuroimage 2002;17: 825-841.

22 Jenkinson M, Beckmann CF, Behrens TE, Woolrich MW, Smith SM: FSL. NeuroImage 2012;62:782-790.

23 Smith SM: Fast robust automated brain extraction. Hum Brain Mapp 2002;17:143-155.

24 Behzadi Y, Restom K, Liau J, Liu TT: A component based noise correction method (CompCor) for BOLD and perfusion based fMRI. Neuroimage 2007;37:90-101.

25 Grabner G, Janke AL, Budge MM, Smith D, Pruessner J, Collins DL: Symmetric atlasing and model based segmentation: an application to the hippocampus in older adults. Med Image Comput Comput Assist Interv 2006; 9(pt 2):58-66.

26 Jenkinson M, Smith S: A global optimisation method for robust affine registration of brain images. Med Image Anal 2001;5:143-156.

27 StataCorp: Stata Statistical Software: Release 11. College Station, StataCorp LP, 2009.

28 Kiebel SJ, Poline JB, Friston KJ, Holmes AP, Worsley KJ: Robust smoothness estimation in statistical parametric maps using standardized residuals from the general linear model. Neuroimage 1999;10:756-766.
29 Friston KJ, Worsley KJ, Frackowiak RS, Mazziotta JC, Evans AC: Assessing the significance of focal activations using their spatial extent. Hum Brain Mapp 1994;1:210-220.

30 Makris N, Goldstein JM, Kennedy D, Hodge SM, Caviness VS, Faraone SV, et al: Decreased volume of left and total anterior insular lobule in schizophrenia. Schizophr Res 2006;83: 155-171.

31 Selles RW, Li X, Lin F, Chung SG, Roth EJ, Zhang LQ: Feedback-controlled and programmed stretching of the ankle plantarflexors and dorsiflexors in stroke: effects of a 4-week intervention program. Arch Phys Med Rehabil 2005;86:2330-2336.

32 Wu CL, Huang MH, Lee CL, Liu CW, Lin LJ, Chen $\mathrm{CH}$ : Effect on spasticity after performance of dynamic-repeated-passive ankle joint motion exercise in chronic stroke patients. Kaohsiung J Med Sci 2006;22:610-617.

33 Zhang LQ, Chung SG, Bai Z, Xu D, van ReyEM, Rogers MW, et al:Intelligent stretching of ankle joints with contracture/spasticity. IEEE Trans Neural Syst Rehabil Eng 2002;10:149-157.

34 Hobart JC, Cano SJ, Zajicek JP, Thompson AJ: Rating scales as outcome measures for clinical trials in neurology: problems, solutions, and recommendations. Lancet Neurol 2007;6:1094-1105.

35 Clarkson HM: Joint Motion and Function Assessment: A Research-Based Practical Guide, ed 6. Philadelphia, Lippincott Williams \& Wilkins, 2005.

36 Ciccarelli O, Toosy AT, Marsden JF, Wheeler-Kingshott CM, Sahyoun C, Matthews PM, et al: Identifying brain regions for integrative sensorimotor processing with ankle movements. Exp Brain Res 2005;166:31-42.

37 Francis S, Lin X, Aboushoushah S, White TP, Phillips M, Bowtell R, et al: fMRI analysis of active, passive and electrically stimulated ankle dorsiflexion. Neuroimage 2009;44:469-479.

38 Belforte G, Eula G: Design of an active-passive device for human ankle movement during functional magnetic resonance imaging analysis. Proc Inst Mech Eng H 2012;226:21-32.

39 Hollnagel C, Brügger M, Vallery $\mathrm{H}$, Wolf $\mathrm{P}$, Dietz V, Kollias S, et al: Brain activity during stepping: a novel MRI-compatible device. J Neurosci Methods 2011;201:124-130.

40 Zimmermann KM, Bischoff M, Lorey B, Stark R, Munzert J, Zentgraf K: Neural correlates of switching attentional focus during finger movements: an fMRI study. Front Psychol 2012;3:555.

41 Adhikari BM, Quinn KM, Dhamala M: Is the brain's inertia for motor movements different for acceleration and deceleration? PLoS One 2013;8:e78055. 